\title{
Differentialdiagnose der Hautveränderungen an den Beinen bei CVI
}

\author{
Barbara Binder
}

Eingegangen: 6. April 2016 / Angenommen: 9. Juni 2016 / Online publiziert: 5. Juli 2016

(c) Der/die Autor(en) 2016. Dieser Artikel ist eine Open-Access-Publikation.

Zusammenfassung Varizen und postthrombotisches Syndrom können bei längerem Bestehen ohne dementsprechende Therapie $\mathrm{zu}$ reversiblen und irreversiblen Hautveränderungen an den unteren Extremitäten führen. Die typischen klinischen Erscheinungsbilder sollten früh erkannt bzw. die möglichen Differentialdiagnosen ausgeschlossen werden.

Schlüsselwörter Chronisch-venöse Insuffizienz · Hautveränderungen · Differentialdiagnosen

Differential diagnosis of skin changes on the lower extremities in chronic venous insufficiency.

Summary Varicous veins and postthrombotic syndrome can make typical reversible or irreversible skin changes on the lower extremities if no treatment is initiated. The typical clinical signs should be recognised in an early stage and possible differential diagnoses have to be excluded.

Keywords Chronic venous insufficiency · Skin changes - Differential diagnoses

Die chronisch-venöse Insuffizienz (CVI) entsteht durch die Dekompensation einer vorbestehenden Venenerkrankungen, das betrifft die Insuffizienz der Venenklappen (Varikose, postthrombotisches Syndrom) und/oder Insuffizienz der zusätzlichen Pumpmechanismen, im speziellen durch Wegfall der GelenksMuskelpumpe. Pathophysiologische Grundlage sind

Priv.-Doz. Dr. B. Binder $(\bowtie)$

Univ.-Klinik für Dermatologie und Venerologie,

Medizinische Universität Graz,

Auenbruggerplatz 8, 8036 Graz, Österreich

E-Mail: barbara.binder@klinikum-graz.at

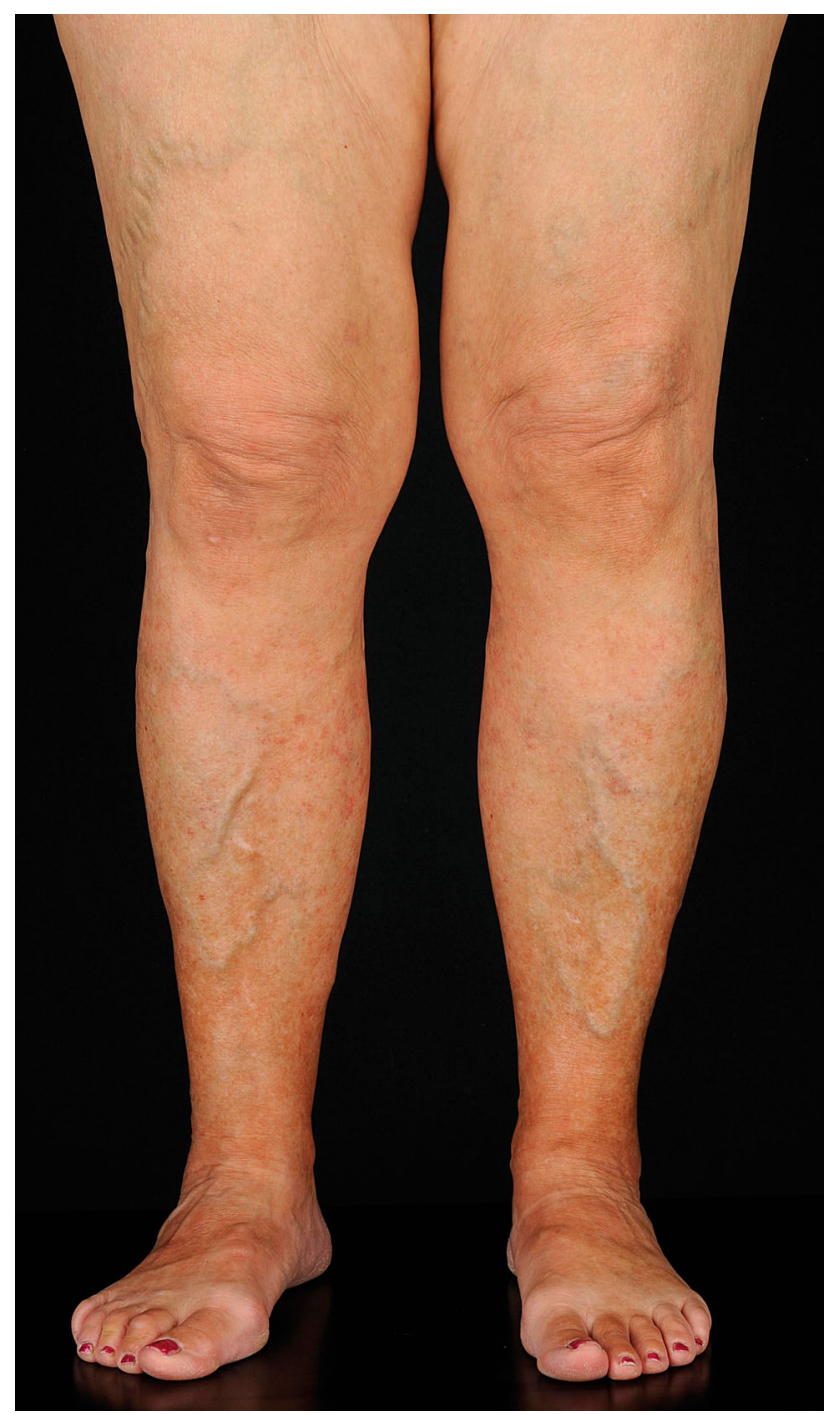

Abb. 1 Purpura jaune d'ocre 

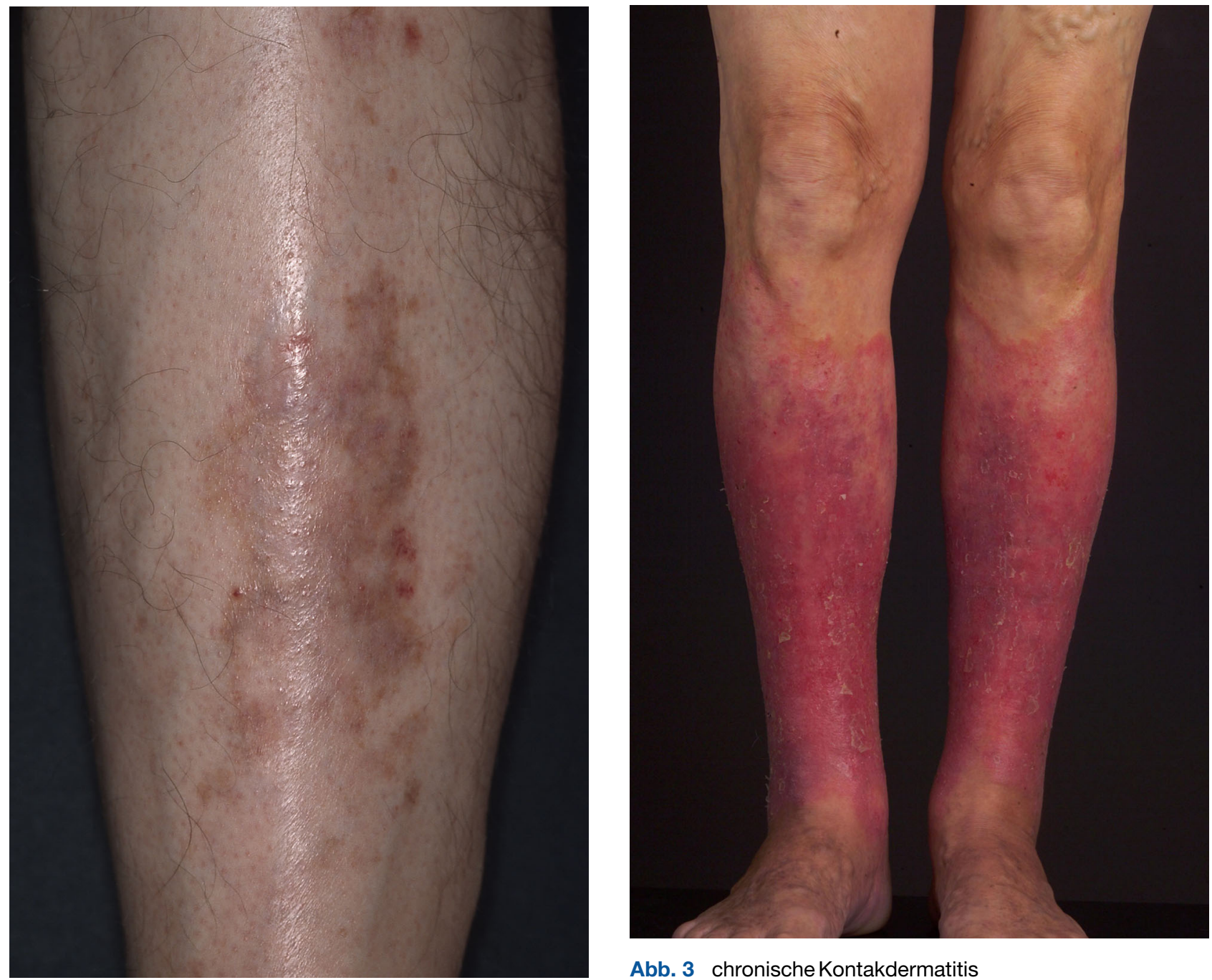

Abb. 3 chronische Kontakdermatitis

Abb. 2 Diabetische Dermopathie

Abb. 4 Necrobiosis lipoidica

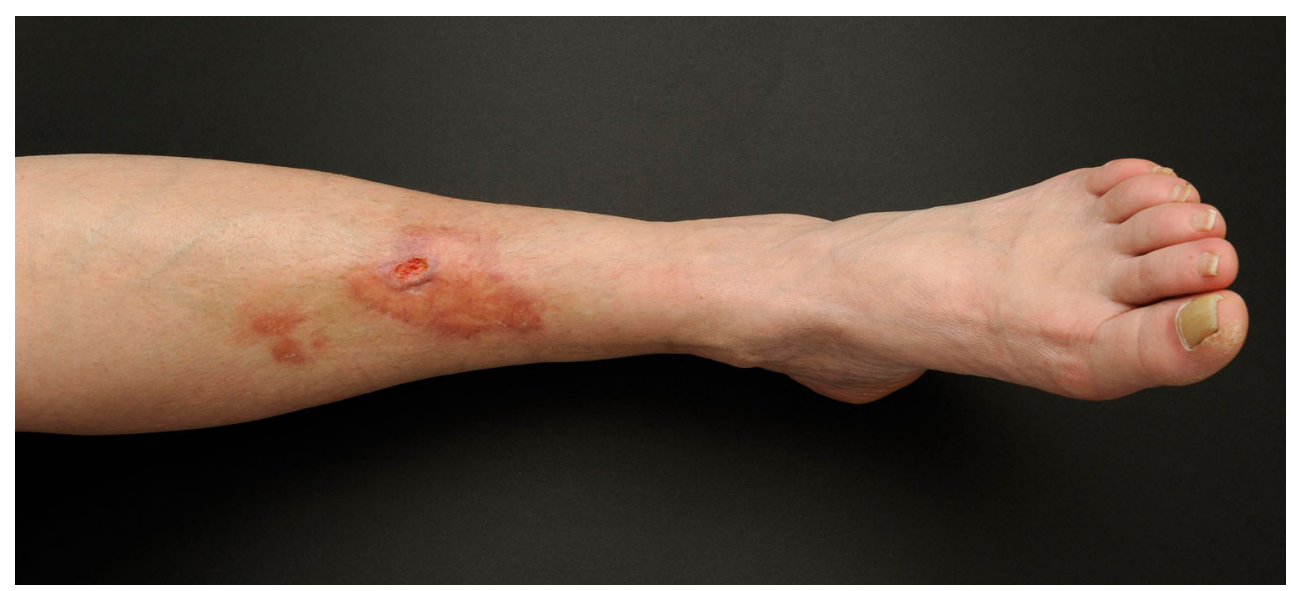



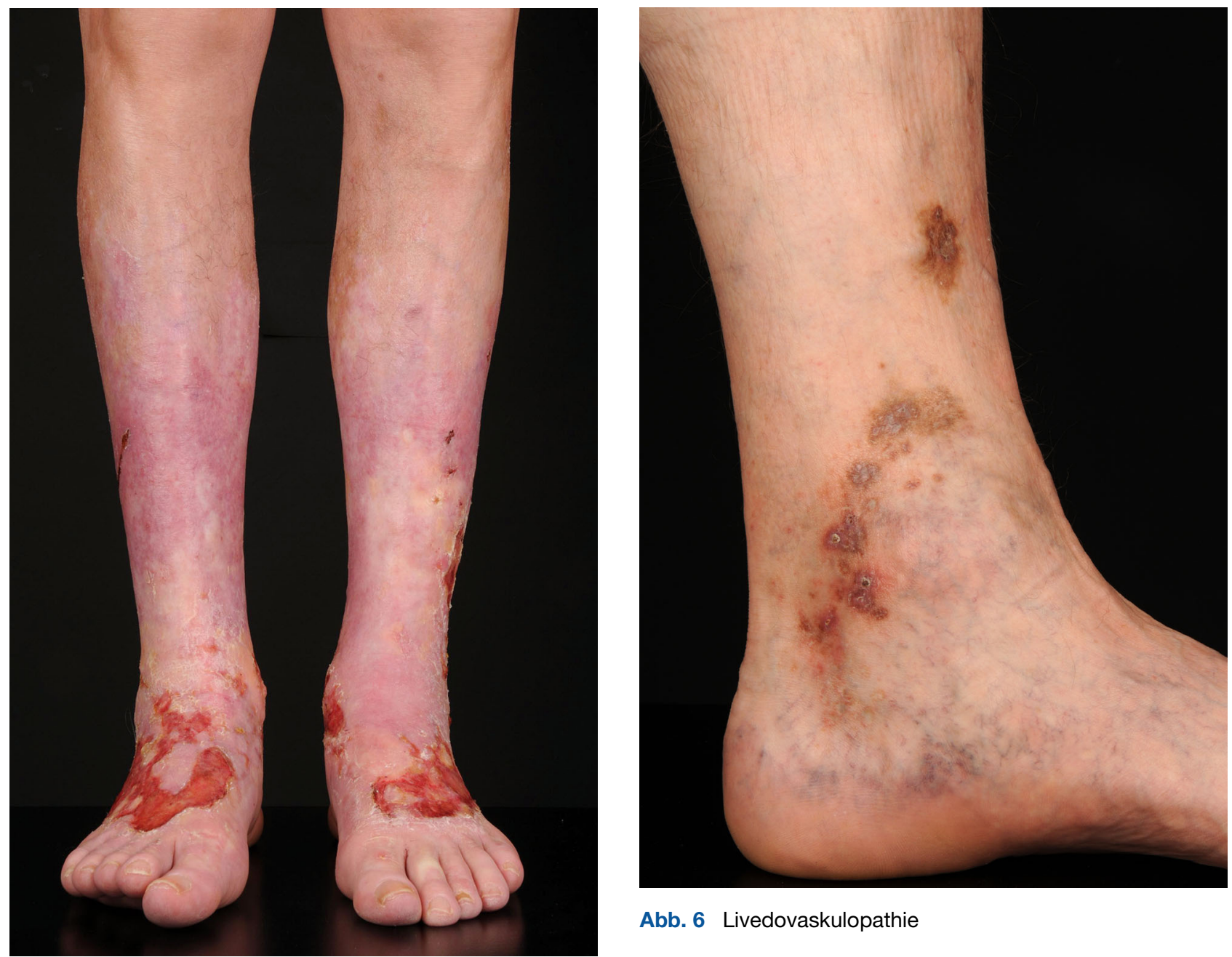

Abb. 6 Livedovaskulopathie

Abb. 5 Zirkumskripte Sklerodermie

Abb. 7 Ulcus cruris venosum und Hypodermitis

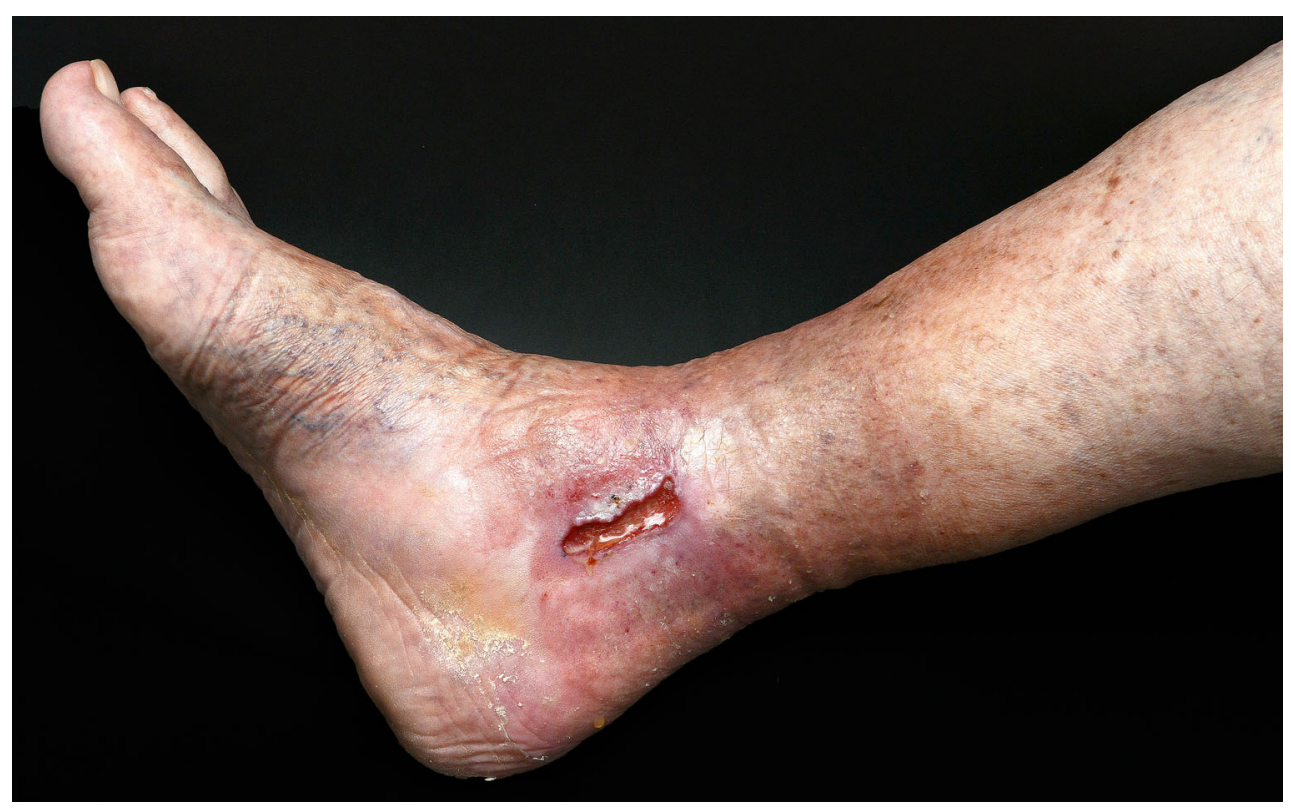




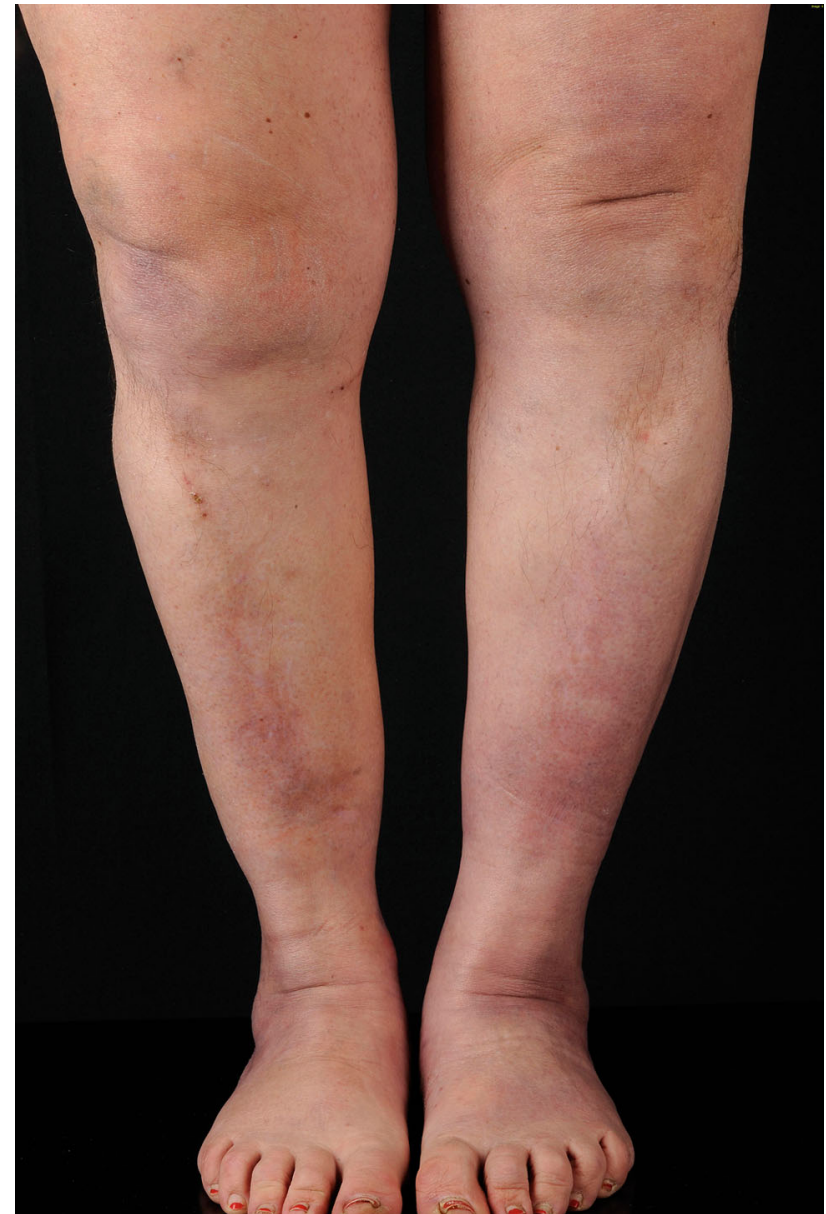

Abb. 8 Acrodermatitis chronica atrophicans

die ambulatorische venöse und kapilläre Hypertonie. Dadurch kommt es zu einer Störung der Mikrozirkulation in der Haut mit konsekutiven Hautveränderungen $[1,2]$. Anhand klinischer Aspekte lässt sich die CVI nach Widmer in drei Grade einteilen:

- Grad 1: Corona phlebectatica paraplantaris (besenreiserartige Venen um die Knöchel und oberhalb des Fußgewölbes), Knöchelödeme.

- Grad 2: Pigmentveränderungen, Ekzemreaktionen, Dermatoliposklerose, Atrophie blanche Herde, Unterschenkelödeme.

- Grad 3: abgeheiltes oder florides Ulcus cruris venosum.

Treten periphere Ödeme auf, müssen auch verschiedenste andere Ursachen bedacht werden, v. a. wenn die Schwellungen beidseitig sind. Eine dementsprechende weitere Abklärung sollte nach Ausschluss einer venösen Komponente durchgeführt werden.

Die Hautveränderungen an den unteren Extremitäten zeigen in den verschiedenen Stadien der CVI charakteristische klinische Bilder, doch sind mögliche Differentialdiagnosen vor der Behandlung zu berücksichtigen [3].

Bei den Pigmentveränderungen kommt es zu fleckförmigen, teils rötlichen, teils rotbraunen oder ocker- farbenen Purpura-Herden in der Umgebung venöser Gefäße (Purpura jaune d'ocre). Abzugrenzen ist sie von einer Purpura pigmentosa progressiva, die unabhängig von einer CVI entstehen kann. Ursächlich können Medikamente, Nahrungsmittelzusatzstoffe, Kryoglobulinämie, Färbe- oder Bleichmittel in Baumwolltextilien, Thrombozytopenien unterschiedlicher Genese als auch post inflammtorische Hyperpigmentierungen dieses klinische Erscheinungsbild auslösen (Abb. 1). Zusätzlich kann es im Rahmen der CVI auch zu flächenhaften Hyperpigmentierungen kommen, sehr ähnlich der Diabetischen Dermopathie (Abb. 2).

Ekzemreaktionen können sowohl akut als auch chronisch auftreten; klinisch präsentieren sie sich als Exsiccations-, Stauungs- oder mikrobielles Ekzem. Vor allem beim mikrobiellen Ekzem sollte eine Epidermomykose mit in die differentialdiagnostischen Überlegungen einfließen. Eine kontaktallergische bzw irritativ- toxische Komponente sollte ebenfalls ausgeschlossen werden (Abb. 3; [4]). Auch kann sich eine Necrobiosis lipoidica hinter dem klinischen Bild eines Ekzems verbergen (Abb. 4).

Bei weiterem Fortschreiten der CVI kommt es zur sog. Dermatosklerose, einer Induration der Subcutis. Bei Fortschreiten können auch das Unterhaugewebe und die Fascien mit einbezogen werden [5]. Ausgeschlossen sollten eine zirkumskripte Sklerodermie (Abb. 5) ebenso wie Pannikulitiden (z. B. bei Lupus erythemtodes bzw. Dermatomyositis) werden.

Die Atrophie blanche ist gekennzeichnet durch weißliche, eingesunkene narbige Areale, welche meist in der Knöchelregion vorkommen. Zu unterscheiden sind diese Areale von postläsionellen Narben bzw. einer Livedovaskulopathie (Abb. 6).

Das Ulcus cruris ist zwar am häufigsten durch eine Venenerkrankung verursacht, doch gibt es auch dabei verschiedenste Differentialdiagnosen $\mathrm{zu}$ bedenken. Diese werden an anderer Stelle diskutiert.

Eine oft verkannte Hauterscheinung bei länger bestehender CVI ist die sog, Hypodermitis. Typisch ist initial eine schmerzhafte Rötung mit Induration. Dies kann sowohl die Knöchelregion als auch die Unterschenkel betreffen. Die Patienten zeigen keine Systemzeichen einer Infektion und die Laborparameter betreffend eine Entzündung sind unauffällig (Abb. 7). Die häufigste Fehldiagnose ist das Erysipel, sodass es immer wieder zur Verabreichung von systemischer antibiotischer Therapie kommt, ohne wirklich therapeutischen Erfolg [6].

Eine seltene, aber unterdiagnostizierte Differentialdiagnose ist die Acrodermatitis chronica atrophicans, eine charakteristische kutane Manifestation der späten Lyme-Borreliose. Im akut-entzündlichen Stadium zeigt sich eine teigige Schwellung mit unscharf begrenzten rötlich-lividen Verfärbungen, daher wird oft die Diagnose CVI gestellt (Abb 8; [7]).

Die chronisch-venöse Insuffizienz führt zu typischen klinischen Erscheinungsbildern an der Haut der unteren Extremitäten [8]. Doch sollten trotzdem 


\section{themenschwerpunkt}

immer auch die möglichen Differentialdiagnosen vor der Behandlung berücksichtigt werden.

Open access funding provided by Medical University of Graz

Interessenkonflikt B. Binder gibt an, dass kein Interessenkonflikt besteht.

Open Access Dieser Artikel wird unter der Creative Commons Namensnennung 4.0 International Lizenz (http:// creativecommons.org/licenses/by/4.0/deed.de) veröffentlicht, welche die Nutzung, Vervielfältigung, Bearbeitung, Verbreitung und Wiedergabe in jeglichem Medium und Format erlaubt, sofern Sie den/die ursprünglichen Autor(en) und die Quelle ordnungsgemäß nennen, einen Link zur Creative Commons Lizenz beifügen und angeben, ob Änderungen vorgenommen wurden.

\section{Literatur}

1. Eberhardt RT, Raffetto JD. Chronic venous insufficiency. Circulation. 2014;130:333-46.
2. Deutsche Gesellschaft für Phlebologie, Rabe E. Leitlinien zur Diagnsotik und Therapie von Venenerkrankungen. Stuttgart New York: Schattauer; 1999.

3. Busch C, Schnabl S. Characteristic skin changes in CVI. Phlebologie. 2014;43:108-11.

4. Lehnen M, Kohaus S, Körber A, Hillen U, Grabbe S, Dissemond J. Konatktsensibilisierungen von Patienten mit chronischen Wunden. Hautarzt. 2006;57:303-8.

5. Miteva M, Romanelli P, Kirsner R. Lipodermatosclerosis. DermatolTher. 2010;23:375-88.

6. Reich-Schupke S, Kreuter A, Altmeyer P, Stücker M. Wrong diagnosis erysipelas: hypodermitis - case series and review of the literature. J Dtsch Dermatol Ges. 2009;7:222-5.

7. Binder B, Kerl H, Müllegger RR. Differenzialdiagnose der Acrodermatitis chronica atrophicans unter besonderer Berücksichtigung der chronisch-venösen Insuffizienz. Phlebologie. 2004;33:191-8.

8. Noppeney T, Nüllen H. Varikose: Diagnostik, Therapie, Begutachtung. Heidelberg: Springer; 2010. 